Revista de Ciencias Sociales - Número 68 (2016) - Páginas 137-154

La imposición estatal para cotizar a la seguridad social y la garantía...

\title{
LA IMPOSICIÓN ESTATAL PARA COTIZAR A LA SEGURIDAD SOCIAL Y LA GARANTÍA DE LA LIBERTAD
}

\author{
THE STATE TAXATION TO SOCIAL SECURITY \\ AND THE WARRANTY OF FREEDOM
}

\author{
RODRIGO MONTEIRO PESSOA* \\ Universidad de la Frontera (Chile) \\ rodrigopessoa@hotmail.cl
}

\section{Resumen}

A lo largo del tiempo el hombre siempre ha tenido preocupación con respecto a su seguridad. Las adversidades de la vida nos llevan a experimentar inquietudes acerca del futuro y las probables contingencias. Debido a la constante preocupación, ha surgido la seguridad social como instrumento regulado por el Estado a través de su coerción, sustituyendo la voluntad de sus ciudadanos para sistematizar la cotización del seguro público. Pero ¿̇cómo

* Profesor de Derecho del Trabajo en la Universidad de la Frontera. Licenciado en Administración en la Universidade Federal da Paraíba-Brasil (2002); Licenciado en Ciencias Jurídicas en el Instituto Superior de Educação da ParaíbaBrasil (2008); Diplomado en Derecho de la Seguridad Social por Uniderp-Brasil (2011); Magíster Académico en Derecho Económico por la Universidade Federal da Paraíba-Brasil (2013); Candidato a Doctor en Derecho por la Universidad de Chile, becado por CONICYT. Temuco, Chile. Artículo recibido el 11 de abril y aceptado el 10 de junio de 2016.

Revista de Ciencias Sociales - Número 68 (2016) - Universidad de Valparáíso - ISSN 0716-7725-Valparáiso, Chile 
se vislumbra la libertad en ese contexto? ¿Es posible hablar de libertad frente a la obligatoriedad de ahorrar para un sistema de seguridad social?

\section{Palabras clave}

Seguridad social, Estado, libertad.

\section{Abstract}

Throughout time man has always had concerns about their safety. The adversities of life lead us to experience concerns about the future and the probable contingencies. Due to the constant concern, social security has emerged as an instrument regulated by the state through its coercion, substituting the will of its citizens to systematize the public insurance quote. But how freedom is seen in that context? Is it possible to speak of freedom from compulsory saving for a social security system?

\section{Keywords}

Social security, State, freedom.

\section{Aproximación histórico de la seguridad social}

Desde que el hombre comenzó a ahorrar alimentos para el día siguiente, por no saber si habría alguna dificultad para obtenerlos nuevamente, empezamos a conocer los orígenes de la seguridad. Obviamente, en la prehistoria no existían organizaciones estructuradas para hablar específicamente de un sistema de seguridad, pero buscar en la primicia de la humanidad conceptos - no tan desarrolladosdel término seguridad permite darse cuenta de lo previsor y visionario que es el hombre, con respecto a su destino y bienestar.

Con la evolución social y económica, la ideología liberal proyectó un régimen de explotación sin límites. Los pobres no tenían cualquier herramienta capaz de promover el ascenso de su calidad de vida; ya que en las constituciones lo que se bosquejaba era solamente un ideal de derechos fundamentales de rasgo negativo (libertad formal, o libertad sin más), de no intervención del Estado, lo que se conoce como libertades públicas.

Facultad de Derecho y Ciencias Sociales - Universidad de Valparaíso - Chile 
Ante este escenario, los trabajadores debían contratar planes de protección a través de seguros privados o mediante el uso de asociaciones creadas por ellos mismos, pero sin ninguna interferencia del Estado, luego sin obligatoriedad de cotizaciones o solidaridad entre las generaciones.

La seguridad social como "sentimiento universal de solidaridad entre los hombres, antes las penetrantes aflicciones de algunos y generosa sensibilidad de muchos" tiene sus raíces en el crecimiento irresponsable del capitalismo moderno. Cuando la depredadora explotación del capital sobre el trabajo ha dejado a los obreros sin cobertura para las adversidades o eventos fortuitos que podrían ocurrir en su vida laboral, el Estado interventor reguló algunas acciones con la normativa posible, con el fin de garantizar a los trabajadores una protección social primitiva (empero efectiva para el momento) en forma de seguro público.

La primera gran intervención estatal en este sentido se ha dado en Alemania en los fines del siglo XIX con el canciller Otto Von Bismarck y las leyes de seguro enfermedad de 1883, seguro accidente del trabajo de 1884 y seguro de invalidez y vejez de $1889^{2}$.

Tampoco se puede olvidar la constitución Mexicana (1917) y la de Weimar en Alemania (1919) que han propuesto los derechos sociales en su cuerpo normativo como una manera de constitucionalizar la protección del trabajador, incluyendo el seguro público.

Por otra parte tenemos en este período el Social Security Act, de 1935, editado en los Estados Unidos como una de las medidas del New Deal, del Gobierno Roosevelt, donde se empleó por primera vez el término "seguridad social".

En Inglaterra, un hito importante es el Plan Beveridge creado en 1941 por el Lord William Henry Beveridge. Este plan tenía como propósito la instauración de un sistema social de prevención de riesgos, tales como la extrema pobreza y la incapacidad para el trabajo. De

1. RUSSOMANO, Mozart: Curso de Previdência Social. Forense, Río de Janeiro, 1978. Pág. 02.

2. TAPIA, Herman Ceballos y RIVAS, Juan Gumucio: Los trabajadores independientes frente a la seguridad social, Editorial Andrés Bello, Santiago de Chile, 1972. Pág. 17.

Revista de Ciencias Sociales - Número 68 (2016) - Universidad de Valparáíso - ISSN 0716-7725-Valparáiso, Chile 
acuerdo a las disposiciones del plan, la seguridad sería prestada desde la cuna a la tumba (Social security from de cradle to the grave). Entre sus principales características tenemos la unificación de los seguros sociales existentes; el establecimiento de la universalidad de la protección social para todos; igual protección para todos los ciudadanos trabajadores; y la forma triple de cotización, con predominio de la cotización estatal.

En seguida, la seguridad social se desarrolló hasta obtener el status de Derecho Humano Universal, puesto que a fines de la segunda guerra se promulgaron los artículos 22 y 25 de la Declaración Universal de los Derechos Humanos de 1948, que disponen lo siguiente:

Artículo 22: Toda persona, como miembro de la sociedad, tiene derecho a la seguridad social, y a obtener, mediante el esfuerzo nacional y la cooperación internacional, habida cuenta de la organización y los recursos de cada Estado, la satisfacción de los derechos económicos, sociales y culturales, indispensables a su dignidad y al libre desarrollo de su personalidad.

Artículo 25: 1. Toda persona tiene derecho a un nivel de vida adecuado que le asegure, así como a su familia, la salud y el bienestar, y en especial la alimentación, el vestido, la vivienda, la asistencia médica y los servicios sociales necesarios; tiene asimismo derecho a los seguros en caso de desempleo, enfermedad, invalidez, viudez, vejez u otros casos de pérdida de sus medios de subsistencia por circunstancias independientes de su voluntad. 2. La maternidad y la infancia tienen derecho a cuidados y asistencia especiales. Todos los niños, nacidos de matrimonio o fuera de matrimonio, tienen derecho a igual protección social.

Existe aún el Convenio número 102 de 1952, relativo a la norma mínima de la seguridad social de la Organización Internacional del Trabajador (OIT), hecho en Ginebra, planteando el derecho al seguro público en otros niveles de intervención, con su reconocimiento internacional.

En Chile la evolución de la seguridad social siguió las mismas disposiciones planteadas precedentemente en el contexto Europeo. Se inició con el desarrollo de mutuales por iniciativas privadas de grupos

Facultad de Derecho y Ciencias Sociales - Universidad de Valparaíso - Chile 
de trabajadores, desde 18 de septiembre de 1853, cuando fue fundada la sociedad mutualista Unión de los Tipógrafos, por el peruano Victorino Laínez, en la capital chilena.

Luego, el 28 de diciembre de 1898 el Congreso Nacional promulga la ley número 1.146 aprobando el seguro social para los empleados públicos, cuyo artículo único asevera:

Los empleados públicos que justificaren haber servido cuarenta años, sin tomar en cuenta abonos, i que hubieren cumplido sesenta $i$ cinco años de edad, podrán jubilarse con una pension anual igual al sueldo integro asignado a sus respectivos empleos, sin necesidad de justificar imposibilidad física o moral.

En 1924 se comienzan a crear los sistemas estatales más organizados, con importante actividad legislativa, en este contexto se promulgó la Ley $\mathrm{N}^{\circ} 4054$ del Seguro Social, la Ley $\mathrm{N}^{\circ} 4055$ de accidentes del trabajo y la Ley $\mathrm{N}^{\circ} 4059$ de indemnización a empleados particulares. La afiliación se daba por tener la condición de trabajador dependiente con contrato, lo que excluía a los independientes del sistema de seguro. De esta forma, se desarrollan en Chile los estándares modernos de seguridad social que hoy comprendemos. Dichos estándares son financiados con aportes obligatorios de los que ejercen actividad laboral, además de los impuestos generales.

Junto con la Ley $\mathrm{N}^{\circ} 4054$ de 1924 —que ha creado la Caja de Seguro Obligatorio- habían diversos regímenes especiales que eran gestionados por sus debidas Cajas de Previsión, de acuerdo con el tipo de trabajador, o sea, no existía uniformidad en el sistema de seguro social Chileno.

La Ley $\mathrm{N}^{\circ} 4054$ de 1924, determinaba en su artículo $1^{\circ}$ la obligatoriedad del seguro de enfermedad y salud para toda persona menor de sesenta y cinco años de edad, que ordinariamente no tuviera otra renta o medio de subsistencia que el sueldo o salario que le pague su patrón, sea éste persona natural o jurídica. Comprendía en su artículo 15 los beneficios de asistencia médica y provisión de todos los medios terapéuticos necesarios que requirieran desde el primer día de la enfermedad; un subsidio en dinero, mientras dure la incapacidad al 
asegurado que tuviere familia que viva a sus expensas; atención profesional de las aseguradas durante el embarazo, parto o puerperio; y además un auxilio igual al 50 por ciento durante las dos semanas que preceden y siguen al parto y de un 25 por ciento en el período posterior prolongado hasta el destete; indemnización por muerte; pensión de invalidez; y una pensión por retiro.

La salud fue desarrollada con la creación del Servicio Nacional de Salud por la Ley $\mathrm{N}^{\circ} 10.383$ de 1952, que lo preveía en su título II. El artículo 62 de la citada ley disponía: "Créase el Servicio Nacional de Salud encargado de la protección de la salud por medio de acciones sanitarias y de asistencia social y atenciones médicas preventivas y curativas".

La Ley $\mathrm{N}^{\circ} 15.386$ de 11 de diciembre de 1963 aprueba la revalorización de las pensiones, estableciendo un importante cambio axiológico en la seguridad, puesto que en su artículo $1^{\circ}$ determinaba compensar el deterioro sufrido por las pensiones de regímenes previsionales a causa de la desvalorización monetaria y mantener sus montos revalorizados. Esta positivación normativa estaba de pleno acuerdo al texto original de la Constitución Política de la República de Chile de 1933, vigente en la fecha, que en los derechos fundamentales clasificados en su artículo 10, número 16, inciso 1 disponía:

Artículo 10 Asimismo, la Constitución asegura a todos los habitantes de la República:

(...)

$16^{\circ}$ El derecho a la seguridad social.

El Estado adoptará todas las medidas que tiendan a la satisfacción de los derechos sociales, económicos y culturales necesarios para el libre desenvolvimiento de la personalidad y de la dignidad humanas, para la protección integral de la colectividad y para propender a una equitativa redistribución de la renta nacional.

En 1979 el Decreto ley $\mathrm{N}^{\circ} 2763$ reorganiza el Ministerio de Salud y crea los Servicios de Salud, el Fondo Nacional de Salud (FONASA), el Instituto de Salud Pública de Chile y la Central de Abastecimiento del Sistema Nacional de Servicios de Salud.

Ya el 12 de febrero de 1990, la Ley N 18.933 crea la Superintendencia de Instituciones de Salud Previsional (ISAPRE), dicta normas

Facultad de Derecho y Ciencias Sociales - Universidad de Valparaíso - Chile 
para el otorgamiento de prestaciones por ISAPRE y deroga el Decreto con Fuerza de Ley $\mathrm{N}^{\circ} 3$ de salud, de 1981.

Pasada la etapa de universalización, Chile experimenta un cambio considerable en su sistema de Seguridad. En efecto, la promulgación del Decreto Ley $\mathrm{N}^{\circ} 3.500$ de 13 de noviembre de 1980 sustituye el antiguo sistema público por uno administrado por las llamadas Administradoras de Fondos de Pensiones (AFP) que poseen su delineación en el título IV, artículo 23 del citado decreto:

Artículo 23.- Las Administradoras de Fondos LEY de Pensiones, denominadas también en esta ley Administradoras, serán sociedades anónimas que tendrán como objeto exclusivo administrar Fondos de Pensiones y otorgar y administrar las prestaciones y beneficios que establece esta ley.

Este nuevo contexto, "aunque se le presentó como Reforma Previsional, no fue sino la sustitución del Antiguo Sistema previsional, basado en el principio solidario, el financiamiento tripartito y el régimen de reparto, por un Nuevo Sistema, basado en la capitalización individual y la administración privada de los Fondos de Pensiones"3.

Lo que se percibe en realidad es una transferencia de administración con cambio del sistema de reparto simple al de capitalización, pero que sigue con la obligatoriedad de cotizar. Así el Estado sigue con el monopolio de la intervención pública con objetivo de insertar el concepto de bienestar social, mediante la preservación de la dignidad de la persona humana en las contingencias sociales.

\section{La extensión de la libertad y el desarrollo como libertad}

La Libertad es un valor que a lo largo de la historia incita a los pensadores para comprender su significado y delineación.

No son pocas las teorías que buscan conocer sus raíces y comprender como la entendemos hoy. Patterson ${ }^{4}$ afirma que las primeras

3. ELTER, Doris: Sistema de A.F.P. chileno: injusticia de um modelo, LOM Ediciones, Santiago de Chile, 1999. Pág. 05.

4. PATTERSON, Orlando: La libertad: La libertad en la construcción de la cultura occidental, Editorial Andrés Bello, Santiago de Chile. Pág. 35.

Revista de Ciencias Sociales - Número 68 (2016) - Universidad de Valparaíso - ISSN 0716-7725-Valparáís, Chile 
personas que tuvieron la inusual idea de ser libres fueron los esclavos, es decir, la libertad habría surgido, según algunos historiadores, como una condición social especial. En este contexto, el autor explica que la sociedad excluyente de antigüedad alejaba a los esclavos de la participación en los organismos comunitarios y así eran reducidos a cosas, mercancías, pertenecientes a sus señores, a quienes deberían respeto, servidumbre y trabajo.

En la sociedad antigua, el esclavo debía su vida y supervivencia a su señor, éste era quien se encargaba de suministrar los elementos básicos para su protección (ya que eran propiedad de los señores), vivienda y trabajo (incluso obligados). La modificación del status de esclavo no producía grandes beneficios. Al contrario, los esclavos no querían esta llamada "libertad" por el hecho de que seguirían excluidos de la sociedad pero ahora sin protección y sin vivienda y trabajo. Libertad no significaba ciudadanía en ese contexto ${ }^{5}$.

De hecho, tener la idea de un concepto no significa incorporarlo como un valor. Según Patterson, "un valor surge, se lo construye socialmente, sólo cuando una masa crítica de personas, o una minoría poderosa, lo comparte, se conduce de modo persistente de acuerdo con él y lo convierte en normativo".

Con estos preludios podemos entender, por tanto, que la libertad es un valor inherente al ser humano y, pese al intento por distinguir entre diferentes "tipos" de libertades, ella es indivisible aun cuando posee diversos planteamientos. Como, por ejemplo, la libertad positiva, definida como el deseo del individuo de ser señor de sus propias acciones e intenciones, ser consciente de sí mismo, como un ser que piensa, quiere y actúa ${ }^{7}$; o libertad negativa, como el concepto de la no injerencia de otros individuos en la actividades de uno $^{8}$, e incluso la libertad personal

5. Ibídem. Pág. 197.

6. PATTERSON, Orlando: ob. cit. Pág. 77.

7. BERLIN, Isaiah: Quatro ensaios sobre a liberdade. Editora Universidade de Brasília, Brasília, 1981. (Traducción de Wamberto Hudson Ferreira). Pág. 142.

8. Ibídem. Pág. 136.

Facultad de Derecho y Ciencias Sociales - Universidad de Valparaíso - Chile 
o aún la libertad cívica. En todos estos planteamientos estamos hablando de aplicaciones prácticas del mismo valor. La libertad sería entonces una en grado, pero no en clase 9 .

Podemos interpretar la actuación de la libertad o aplicarla en situaciones prácticas; tales como la libertad de expresión, de pactos, de ejercer un oficio, de elegir y ser votado, de acceso al poder judicial o a las políticas públicas, pero en todas estas propuestas estamos delante un valor indivisible. Hablando de esta manera puede incluso parecer que la libertad es un axioma absoluto, sin restricciones, pero no ha sido y nunca será así.

Esto es porque ella es ejercida a partir de un contexto social. La libertad existe en relación a algo y no aisladamente (es comparativa). En esta línea, Kant ya afirmaba que en la presencia del Estado la libertad de cada uno está limitada por el derecho, para que sea de acuerdo con la libertad de todos los demás ${ }^{10}$. De ahí la afirmación de que no es irrestricta, sino coexistente y comunitaria, flexibilizándose de acuerdo al ejercicio de cada uno, para que nadie deje de ser libre en contraste con la absoluta libertad del otro. Las situaciones cotidianas requerirán siempre que alguien avance o renuncie en su derecho de actuar, pensar, hacer y disfrutar por la pacificación de las relaciones intersubjetivas.

Esta práctica restrictiva es del todo interesante, porque las acciones de los hombres serán siempre direccionadas a sus fines egoístas. Lo que mueve la acción humana son sus sentimientos y convicciones, y de esta manera toda energía utilizada para tener éxito en algo viene motivada por su individualidad. Tenemos entonces en Mill que "la única finalidad que justifica la interferencia de los hombres, individual y colectiva, es la libertad de acción de los demás, es la autoprotección" ". Las acciones para que justifiquen una intervención del estado en la esfera particular

9. HAYEK, Friedrich: Los fundamentos de la libertad. $7^{\text {a }}$ edición, Unión editorial, Madrid, 2006. Pág. 33.

10. BRAVO, Edgar et al: Moralidad y Eticidad. Estudios sobre Kant y Hegel, Cali, Editorial Universidad del Valle, 2004. Pág. 131.

11. MILL, John Stuart: Sobre a liberdade. $2^{\mathrm{a}}$ edição, Editora Vozes, Río de Janeiro, 1991. (Traducción Alberto da Rocha Barros). Pág. 53.

Revista de Ciencias Sociales - Número 68 (2016) - Universidad de Valparáíso - ISSN 0716-7725-Valparaíso, Chile 
deben venir acompañadas de perjuicios, propio, de otro u otros. "Si no existe perjuicio o lesividad, no puede haber limitación en la esfera de la libertad privada de la persona"12.

No estamos necesariamente afirmando que el hombre es malo por naturaleza, sólo estamos de acuerdo con la comprensión de Freund cuando alude a las decisiones de los hombres en un contexto de libertad y su ejercicio — proponiendo como objetivo de la política proteger a los miembros de una colectividad particular, de la violencia recíproca incluyendo la del poder, para que todos puedan cumplir su destino según su propio genio, sus gustos y aspiraciones- dice:

Se l'uomo non è né buono né cattivo per natura ed è attraverso le sue scelte e gli usi delle sue scelte che egli utilizza bene o male gli strumenti e le forze a sua disposizione, bisogna allora porre il potere nella stessa rubrica. Tutto dipende dalla maniera in cui lo si esercita. Esso non è ontologicamente buono o cattivo, può però divenire l'una o l'altra cosa secondo le circonstanze $e^{13}$.

Los derechos inherentes al ser humano evolucionaron al mismo nivel que la sociedad y el Estado. La propuesta de Hayek es interesante en este sentido, al posicionar las estructuras sociales como una civilización generada por la experiencia y por el conocimiento, no al extremo del darwinismo social, ni de la libre imaginación creativa del hombre, tampoco en la abstracta teoría contractualista, sino como una mejora según las buenas experiencias, la eliminación de las malas y la aplicación de los conocimientos.

12. ROJAS LABRA, Nicoll y VALDÉS AGUAYO, Diana: “Constitución, Orden Público y Tendencias Legislativas Recientes". En: Revista de Ciencias Sociales, Facultad de derecho, Universidad de Valparaíso, Nº 62, 2013. Págs. 13-37.

13. FREUND, Julien: La crisi dello Stato tra decisione e norma, Alfredo Guida Editora, Nápoli, 2008. Pág. 138. Si el hombre no es ni bueno ni malo por naturaleza y es a través de sus decisiones y usos de sus opciones que él utiliza bien o mal los instrumentos y las fuerzas a su disposición, debe poner entonces el poder en la misma lista. Todo depende de la forma por la cual lo ejercita. Esto no es ontológicamente bueno o malo, sin embargo, puede ser una cosa u otra, dadas las circunstancias (traducción del autor).

Facultad de Derecho y Ciencias Sociales - Universidad de Valparaíso - Chile 
De ahí el Estado evoluciona, se desarrolla y puede ser el encargado de la fijación de los límites de la libertad a través de la fuerza, puesto que "la sociedad libre se ha enfrentado a este problema confiriendo al Estado el monopolio de la coacción, e intentando limitar ese poder estatal a los casos que sea necesario ejercer e impidiendo que dicha coacción la ejerzan personas privadas"14.

Por supuesto que las personas no retrocederían delante a situaciones contrarias a sus intereses, es necesario una tercera parte neutra que actúe en beneficio de la colectividad, capaz de superponer su actuación contra los individuos. Este tercero es el Estado. Y cuando actúa por la colectividad no hay interés particular, sino la voluntad colectiva, por lo que hay una evaluación de lo que es mejor para el bien común contra el bien particular. Al juzgar la relación intersubjetiva, la entidad estatal se esmera por el bien común, donde la mayoría de la gente gana en libertad, más que un individuo únicamente en su ejercicio.

Sin embargo, esto no se dio a través de un contrato social, el estado de naturaleza apuntado por los contractualistas fue desdeñoso incluso por Rousseau en su segundo discurso donde asevera: "Pues no es empresa liviana el deslindar lo que hay de originario y de artificial en la naturaleza actual del hombre, y conocer bien un estado que ya no existe, que tal vez nunca ha existido, que probablemente no existirá jamás y del que no obstante es necesario tener nociones precisas para juzgar bien sobre nuestro estado presente"15.

Además, se sabe que la teoría contractualista no sirve como base para la coerción estatal ya que no hay libertad en la elección de no unirse a este contrato hipotético, y la adhesión requiere voluntad, lo que no existe bajo un ordenamiento jurídico ${ }^{16}$.

14. FREUND, Julien: ob. cit. Pág. 46.

15. ROUSSEAU, Jean Jacques: Discurso sobre el origen y los fundamentos de la desigualdad entre los hombres. En: 'Escritos de combate', Alfaguara, Madrid, 1979. (Traducción Salustiano Masó). Pág. 142.

16. TABORDA, Maren et al: Programa de hermenêutica jurídica: estudos em homenagem a Sandro Subtil da Silva, EDIPUCRS, Porto Alegre, 2011. Pág. 204.

Revista de Ciencias Sociales - Número 68 (2016) - Universidad de Valparáíso - ISSN 0716-7725-Valparáiso, Chile 
El hombre siempre vivió en sociedad y la mejora de la vida en la sociedad es lo que originó el Estado, una reunión de un grupo específico para el bien común a través de una coordinación de esfuerzos y una cooperación mutua organizada ${ }^{17}$. Es decir, no surgió de un pacto para abandonar el estado de la naturaleza, sino de una evolución empleada de esfuerzos y de la experiencia de la razón humana.

Se puede resumir lo planteado hasta ahora en el siguiente silogismo: la libertad es un valor unitario en cuanto género que permite la libre acción humana en la búsqueda de su destino, pero por el hecho de no ser absoluta el Estado es responsable por la demarcación del rendimiento de este valor, luego el Estado establece el paradigma relacional de la libertad en su propósito que es el bien común. Y a menudo esto puede ser también individual si, a través de la coerción, el ente público obliga el ciudadano a realizar acciones que no haría voluntariamente, para que pueda disfrutar de otros bienes disponibles en la vida comunitaria.

En este sesgo la libertad sólo tiene razón de ser si busca el fomento de oportunidades para las personas. La capacidad de generar opciones dentro del universo en que se vive es generar un desarrollo libertario.

Desde la perspectiva de Amartya Sen, el desarrollo debe tener en la libertad el fin y los medios para su concreción. Las riquezas en sí mismo de nada servirían, dice Aristóteles en la Ética Nicomáquea que no es el bien que buscamos, siendo la riqueza solamente útil y en el interés de otra $\operatorname{cosa}^{18}$. Luego, la libertad en el sentido de desarrollo se encuentra en la delimitación de oportunidades disponibles para los sujetos.

En la concepción del desarrollo como libertad, tenemos que examinar en qué grado las personas tienen la oportunidad de obtener resultados que valoran y que tienen razón en valorar. Y eso se pone en práctica en las libertades políticas que son las oportunidades que tienen

17. AZAMBUJA, Darcy: Teoria Geral do Estado, Editora Globo, São Paulo, 2001. Pág. 03.

18. ARISTOTLE: The Nicomachean Ethics, UK: Filiquarian Publishing, LLC, 2007. Pág. 10.

Facultad de Derecho y Ciencias Sociales - Universidad de Valparaíso - Chile 
las personas para determinar quién debe gobernar, supervisar y criticar a las autoridades, la libertad de expresión política, libertad de elegir entre diversos partidos políticos, etc.; facilidades económicas que se determinan en las oportunidades que los individuos tienen para utilizar recursos económicos con el fin de consumo, producción y cambio, siempre mirando el tema de la distribución de los ingresos; oportunidades sociales que son disposiciones que la sociedad establece en las áreas de educación, salud, etc., las cuales influyen la libertad sustantiva del individuo para vivir mejor; garantías de transparencia que se refieren a las necesidades de sinceridad que las personas pueden esperar: libertad para hacer frente los unos a los otros bajo garantías de sinceridad y transparencia; y contando con una red de seguridad protectora, previniendo que la población afectada se reduzca a la miseria extrema y, en algunos casos, protegiéndolos del desamparo, el hambre y la muerte ${ }^{19}$.

Ante la situación hasta entonces expuesta, la pregunta que intentaremos responder en el contexto de este valor humano universal es ¿el Estado en el uso del poder coercitivo para la cotización de la seguridad social trata de limitar la libertad individual de las personas?

Amartya Sen estaba en lo correcto al decir que la libertad debe ser fin y medio del desarrollo, es decir, que sólo se puede lograr el desarrollo si la libertad fue plenamente ofrecida a las personas para que dispongan de aquello que valoran, y el propio desarrollo sólo tiene razón de ser si fuera un mecanismo responsable por alcanzar más libertades instrumentales. Si funciona de esta forma en la evolución social en su conjunto podemos hablar concretamente en el acceso y expansión del axioma en análisis.

La coerción estatal está totalmente justificada desde el momento en que se hace necesaria para regular la convivencia humana. Esta coerción es un elemento jurídico de la formación del Estado y que lo legitima a actuar con la fuerza física o moral en la pacificación social. Pero esta limitación coercitiva del Estado no es arbitraria, usurpadora y sin límites. "Padecer la realidad o la expectativa de la interferencia arbitraria

19. SEN, Amartya: Desenvolvimento como liberdade, Companhia das Letras, São Paulo, 2000. (Traducción Laura Teixeira Motta). Págs. 55-57.

Revista de Ciencias Sociales - Número 68 (2016) - Universidad de Valparáíso - ISSN 0716-7725-Valparaíso, Chile 
es padecer un mal que rebasa con mucho el de ver estorbadas intencionalmente nuestras elecciones. Es tener que soportar un alto nivel de incertidumbre, pues el fundamento arbitrario en que descansa esa interferencia significa que no puede predecirse cuándo nos va a acometer. Esa incertidumbre hace mucho más difícil la planificación que en el caso de la interferencia no-arbitraria. Y, claro está, tiende a generar altos niveles de ansiedad" ${ }^{\prime 2}$.

La no interferencia estatal podría dejar las personas a la suerte de dominación por otros más poderosos, y esta dominación o la mera posibilidad generarían las incertidumbres propuestas por Philip Pettit. Esto es debido al compromiso de la República de Chile para promover el bienestar y la dignidad de las personas. Prontamente, si se sabe que los ciudadanos voluntariamente no ahorrarían sus recursos para una jubilación satisfactoria, y por lo tanto, les llevaría a la miseria, el Estado toma las riendas de la planificación y garantiza la libertad futura.

Obviamente las corrientes más liberales afirmarán que la coerción del ente público con el objetivo de amparar a los necesitados violaría el liberalismo en perspectivas económicas y políticas, pero hay que recordar que el pueblo chileno eligió vivir el constitucionalismo, que impone un paradigma de actuación -adyacente a otros principios que deben ser asegurados junto a la libre iniciativa económica, tales como la solidaridad, la dignidad humana y los valores sociales del trabajo.

Nunca es demasiado recordar que el sistema de Administradoras de Fondos de Pensión, permite elegir la AFP que mejor le parezca al cotizante, sea por su rentabilidad, atención u otras cuestiones relevantes para la contratación, además de permitir en qué tipo de fondo invertir (el más riesgoso, pero con mayor rentabilidad; o el menos riesgoso y con menor rentabilidad; en una escala variable de A a E). Tampoco este sistema impide a que los trabajadores hagan otros tipos de inversión; como cuentas ahorro, inversión en bienes raíces y tantos otros más que son posibles en una sociedad democrática, preservando la libertad en un paradigma de dignidad sin que sea violado su núcleo esencial como derecho fundamental.

20. PETITT, Philip: Republicanismo: una teoría sobre la libertad y el gobierno. Ediciones Paidós Ibérica, Barcelona, 1999. (Traducción Toni Domènech). Pág. 119.

Facultad de Derecho y Ciencias Sociales - Universidad de Valparaíso - Chile 
Así, llegamos a la respuesta de la cuestión propuesta, que en resumen asevera la legalidad de las medidas coercitivas del ente público en la promoción del sistema de seguro social chileno, en reemplazo a la voluntad individual como promoción de la libertad en su sentido más amplio.

Sólo con esta imposición se puede alejar a los individuos de la escasez y garantizar al menos las condiciones mínimas de supervivencia, que les asegure seguir eligiendo a los bienes de la vida que tienen motivo estimar (subjetivamente) y proporcionando el bien común.

\section{Conclusiones}

El seguro es una antigua preocupación en la sociedad. Protegerse de las adversidades y contingencias hicierón que el hombre desarrollara a lo largo del tiempo una forma de disminuir las incertidumbres de la vida.

Desde el momento en que hay una organización como comunidad global y se propone sobre esta organización la figura del Estado, nada más justo que dejarle la responsabilidad de regular y proporcionar el bienestar colectivo primando sobre el interés individual.

Con todo este panorama, se puede verificar que en Chile el actual sistema de seguridad social es un factor mínimo de sobrevivencia, pero que garantiza igualmente la existencia digna del ser humano a través de la salud, asistencia social y previsión.

La adopción de este sistema de seguridad social es un perfeccionamiento de la libertad. Bajo esta perspectiva se ofrece la instrumentalización de este derecho fundamental que junto a la dignidad humana amplía el alcance de la libertad en cuanto género. Libertad que es comprensible en cuanto especie.

Cuando la República de Chile impone la cotización del seguro estatal como obligatorio en sustitución a la voluntad particular, ella no invade la esfera de actividad de los ciudadanos. Opuestamente, por saber que ellos no lo harían de libre aspiración, garantiza el mismo acceso a las opciones de bienes en el futuro a través de la cobertura de contingencias más crueles y debilitantes.

Este trabajo no tiene por objetivo entrar en la discusión sobre la rentabilidad de las AFP, la ganancia con el préstamo de las cotizaciones,

Revista de Ciencias Sociales - Número 68 (2016) - Universidad de Valparáíso - ISSN 0716-7725-Valparaíso, Chile 
la fiscalización del gobierno en la gestión de estos órganos, ni la justicia social de las pensiones. Lo que se hizo hincapié fue tan sólo confirmar la justicia social de la preservación de la dignidad humana delante las contingencias que pueden tomar la fuerza laboral del individuo, llevándolo a la indigencia si no tuviera ningún tipo de seguro.

Esta es la verdadera libertad, que permite el desarrollo y que la tiene como finalidad. Que llega a suministrar las opciones y amplía el rol electivo del hombre. Que proporciona su dignidad y su ciudadanía, es decir, que camina junto a varios otros valores, distanciándolos de la opresión, de la coacción autoritaria, de la esclavitud y de la sumisión exacerbada al capricho ajeno. Sin embargo, no es una libertad absoluta, ya que debe coexistir con varias otras libertades dentro del mismo contexto social. Dilatando o comprimiéndola de acuerdo con el caso específico, sin aislar a alguien de este mismo valor ni dejar que otros vengan a ejercerlo excesivamente.

Indudablemente, con estas líneas no se puede concluir otra cosa que la existencia de la promoción de la libertad por el sistema de seguro social que logra obtener la valoración del ser humano y prestarle una reducción de las incertidumbres, dejándole libre para emplear sus esfuerzos en el logro de sus objetivos personales, sabiendo estar apoyado por un sistema funcional que le permitirá aliviarse cuando esté afligido por un imprevisto, sin que con eso se pierda en plenitud la posibilidad de elección.

\section{Bibliografía}

ARISTÓTELES: Política. Ediciones Istmo, Madrid, 2005. (Edición de Pedro López Baja de Quiroga y Estela García Fernández).

ARISTOTLE: The Nicomachean Ethics. UK, Filiquarian Publishing, LLC, 2007.

AZAMBUJA, Darcy: Teoria Geral do Estado. Editora Globo, São Paulo, 2001.

BALERA, Wagner: Direito previdenciário: série concursos públicos. Editora Método, São Paulo, 2009.

BERLIN, Isaiah: Quatro ensaios sobre a liberdade. Editora Universidade de Brasília, Brasília, 1981. (Traducción Wamberto Hudson Ferreira).

Facultad de Derecho y Ciencias Sociales - Universidad de Valparaíso - Chile 
BRAVO, Edgar et al, Moralidad y Eticidad. Estudios sobre Kant y Hegel. Cali, Editorial Universidad del Valle, 2004.

ELTER, Doris: Sistema de A.F.P. chileno: injusticia de un modelo. LOM Ediciones, Santiago de Chile, 1999.

FREUND, Julien: La crisi dello Stato tra decisione e norma. Alfredo Guida Editora, Nápoli, 2008.

GIRÓN, Jesús; VARELA, Alberto y VÁZQUEZ, Xosé: Derecho de la seguridad social. Segunda Edición, Netbiblo, La Coruña (España), 2008.

HAYEK, Friedrich: Los fundamentos de la libertad. $7^{\mathrm{a}}$ edición, Unión Editorial, Madrid, 2006.

NOGUER, Héctor: Derecho del Trabajo y de la Seguridad Social. Tomo III, Reimpresión de la $17^{a}$ edición, Editorial Jurídica de Chile, Santiago de Chile, 2012.

NOVOA FUENZALIDA, Patricio: Derecho de Seguridad Social. Editorial Jurídica de Chile, Santiago de Chile. 1977.

MILL, John Stuart: Sobre a liberdade. 2a edição, Editora Vozes, Río de Janeiro, 1991. (Traducción Alberto da Rocha Barros).

PATTERSON, Orlando: La libertad: La libertad en la construcción de la cultura occidental. Editorial Andrés Bello, Santiago de Chile, 1991.

PETITT, Philip: Republicanismo: una teoría sobre la libertad y el gobierno.

Ediciones Paidós Ibérica, Barcelona, 1999. (Traducción Toni Domènech).

ROJAS LABRA, Nicoll y VALDÉS AGUAYO, Diana: "Constitución, Orden Público y Tendencias Legislativas Recientes”. En: Revista de Ciencias Sociales, Facultad de derecho, Universidad de Valparaíso, $\mathrm{N}^{\circ}$ 62, 2013. Págs. 13-37.

ROUSSEAU, Jean Jaques: Discurso sobre el origen y los fundamentos de la desigualdad entre los hombres. En: 'Escritos de combate'. Alfaguara, Madrid, 1979. (Traducción Salustiano Masó).

RUSSOMANO, Mozart: Curso de Previdência Social. Forense, Río de Janeiro, 1978.

SEN, Amartya: Desenvolvimento como liberdade. Companhia das Letras, São Paulo, 2000. (Traducción Laura Teixeira Motta). 
TABORDA, Maren et al: Programa de hermenêutica jurídica: estudos em homenagem a Sandro Subtil da Silva. EDIPUCRS, Porto Alegre, 2011.

TAPIA, Herman y RIVAS, Juan: Los trabajadores independientes frente a la seguridad social. Editorial Andrés Bello, Santiago de Chile, 1972.

\section{NORMAS CITADAS}

Convenio $N^{\circ} 102$ de la OIT de 28 de junio de 1952, Relativo a la norma mínima de la seguridad social de la OIT, hecho en Ginebra.

Declaración Universal de los Derechos Humanos de 1948. Asamblea General de las Naciones Unidas. Resolución 217 A (III) del 10 de diciembre de 1948. París.

Decreto Ley N 3.500 de 13 de noviembre de 1980, Que establece un nuevo sistema de pensiones. Diario Oficial de Chile, $13 \mathrm{de}$ noviembre de 1980 .

Decreto $\mathrm{N}^{\circ} 3.048$ de 6 de mayo de 1999, Aprueba el Reglamento de Seguridad Social y más. Diario Oficial de Brasil, 07 de mayo de 1999.

Ley $\mathrm{N}^{\circ} 18.469$ de 14 de noviembre de 1985, Regula el ejercicio del derecho constitucional a la proteccion de la salud y crea un régimen de prestaciones de salud. Diario Oficial de Chile, 23 de noviembre de 1985.

Ley $\mathrm{N}^{\circ} 20.255$ de 11 de marzo de 2008, Establece la reforma previsional. Diario Oficial de Chile, 17 de marzo de 2008.

Ley No 4.054 de 08 de septiembre de 1924, Crea la Caja de Seguro Obligatorio. Diario Oficial de Chile, 26 de septiembre de 1924. Ley $\mathrm{N}^{\mathrm{O}} 4.055$ de 28 de mayo de 1931, Que reforma las leyes de accidentes del trabajo. Diario Oficial de Chile, 26 de septiembre de 1924. Ley $\mathrm{N}^{\circ} 4.059$ de 16 de septiembre de 1924, Instala reglas sobre el contrato de trabajo entre patrones y empleados particulares y contiene disposiciones sobre indemnización por enfermedades o discapacidades laborales y profesionales. Diario Oficial de Chile, 27 de septiembre de 1924.

Facultad de Derecho y Ciencias Sociales - Universidad de Valparaíso - Chile 